\title{
A bifurcation analysis of two coupled calcium oscillators
}

\author{
Michael Bindschadler \\ Department of Mathematics, University of Michigan, Ann Arbor, Michigan \\ James Sneyd ${ }^{\mathrm{a})}$ \\ Institute of Information and Mathematical Sciences, Massey University Albany Campus, \\ Private Bag 102-904, North Shore Mail Centre, Auckland, New Zealand
}

(Received 5 July 2000; accepted for publication 28 November 2000)

\begin{abstract}
In many cell types, asynchronous or synchronous oscillations in the concentration of intracellular free calcium occur in adjacent cells that are coupled by gap junctions. Such oscillations are believed to underlie oscillatory intercellular calcium waves in some cell types, and thus it is important to understand how they occur and are modified by intercellular coupling. Using a previous model of intracellular calcium oscillations in pancreatic acinar cells, this article explores the effects of coupling two cells with a simple linear diffusion term. Depending on the concentration of a signal molecule, inositol $(1,4,5)$-trisphosphate, coupling two identical cells by diffusion can give rise to synchronized in-phase oscillations, as well as different-amplitude in-phase oscillations and same-amplitude antiphase oscillations. Coupling two nonidentical cells leads to more complex behaviors such as cascades of period doubling and multiply periodic solutions. This study is a first step towards understanding the role and significance of the diffusion of calcium through gap junctions in the coordination of oscillatory calcium waves in a variety of cell types. (C) 2001 American Institute of Physics. [DOI: 10.1063/1.1342161]
\end{abstract}

Waves of increased free calcium concentration are one important mechanism whereby cells can coordinate their behavior with that of their neighbors, and are seen in a variety of cell types. Often such waves travel from cell to cell in an oscillatory manner, thus forming periodic intercellular calcium waves; it is the period of these waves that is thought to control and coordinate a variety of cellular processes. The mechanisms underlying such periodic intercellular waves are not well understood in any cell type. Using a model based upon the intracellular calcium dynamics of pancreatic acinar cells, we study the behavior of two oscillating cells that are coupled by the diffusion of calcium through gap junctions. Using numerical methods to study the bifurcation structure of the coupled equations we find that a multitude of periodic behaviors arise, including in-phase oscillations, anti-phase oscillations, multiply-periodic orbits, and oscillations of different amplitudes. Our results provide a basis for the detailed study of coupled calcium oscillators, a necessary first step for the understanding of periodic intercellular calcium waves.

\section{INTRODUCTION}

Intercellular calcium waves are observed in a variety of cell types ${ }^{1,2}$ and occur by a variety of mechanisms. In epithelial cell cultures, for instance, a mechanically stimulated intercellular calcium wave appears to propagate via the intercellular diffusion of the messenger inositol (1,4,5)trisphosphate $\left(\mathrm{IP}_{3}\right)$ which releases $\mathrm{Ca}^{2+}$ from the endoplas-

a) Author to whom correspondence should be addressed; electronic mail: j.sneyd@massey.ac.nz mic reticulum. ${ }^{3}$ In hepatocytes, oscillatory intercellular $\mathrm{Ca}^{2+}$ waves occur in response to vasopressin, ${ }^{4-6}$ and these appear to be generated by the coupling of intracellular $\mathrm{Ca}^{2+}$ oscillations. ${ }^{7,8}$ Oscillatory intercellular calcium waves also occur in the blowfly salivary gland $;^{9}$ a plausible hypothesis is that these waves are the result of intercellular coupling by means of the diffusion of $\mathrm{Ca}^{2+}$ through gap junctions.

There are, in general, two mechanisms by which oscillatory intercellular calcium waves may occur. First, if each cell is able to respond to an increase in intracellular calcium (say through gap junctions from a neighboring cell) by the release of a large amount of additional calcium from internal stores, then a field of connected cells could form an excitable medium, able to propagate a traveling wave of calcium in an active manner. Then, given a pacemaker cell oscillating with sufficient amplitude, each oscillation of the pacemaker could send out a traveling wave, thus resulting in a series of waves traveling across the field of cells, i.e., an oscillatory intercellular calcium wave. Such a mechanism is relatively easily modeled, and has been done so before. ${ }^{10}$

Second, if each cell is an autonomous oscillator but is coupled to its neighbors by the diffusion of a signal molecule (either through gap junctions or extracellularly), then the individual oscillations can be coordinated by the coupling into periodic waves that traverse the field of cells. In pancreatic acinar cells and hepatocytes there is very good evidence that each cell is an autonomous oscillator, and the periodic intercellular waves are the result of this second mechanism. 5,6,11 However, although this hypothesis seems plausible, it is a nontrivial matter to demonstrate that, given the known cell parameters and calcium kinetics, diffusion of calcium through gap junctions can result in a coordinated oscillatory intercellular wave that lasts for many cycles, and extends 


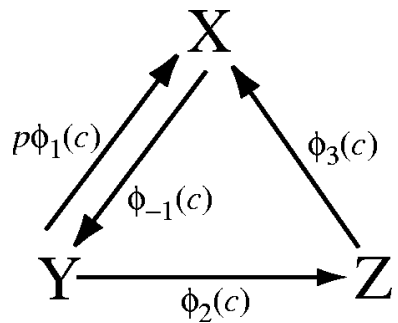

FIG. 1. Schematic diagram of the receptor model.

over distances that are large with respect to the size of an individual cell.

In this article we do not come anywhere near a complete resolution of this question, but study instead a more restricted problem. In order to understand the mechanisms underlying the propagation of oscillatory intercellular calcium waves it is necessary first to understand how intercellular coupling affects the calcium oscillations in each individual cell. As a first approach to this question, here we study the dynamic behavior of two spatially homogeneous cells, coupled by the diffusion of calcium.

\section{THE MODEL EQUATIONS}

The intracellular calcium dynamics are described by the model of LeBeau et al., ${ }^{12}$ a model that was designed particularly to model calcium oscillations in pancreatic acinar cells. Pancreatic acinar cells have a fairly simple spatial arrangement (ringed around a central duct), and the calcium wave travels from cell to cell around the duct in a characteristic manner. ${ }^{11}$ Acinar cells are not electrically excitable, and the calcium causing the increased concentration is released from intracellular stores (the endoplasmic reticulum, or ER). The intracellular signal for this release is inositol $(1,4,5)$ trisphosphate $\left(\mathrm{IP}_{3}\right)$, which triggers $\mathrm{Ca}^{2+}$ release by binding to a calcium channel (the $\mathrm{IP}_{3}$ receptor) on the surface of the ER. This opens the channel, allowing $\mathrm{Ca}^{2+}$ to flow out into the cytoplasm. Subsequent inactivation of the $\mathrm{IP}_{3}$ receptors and removal of $\mathrm{Ca}^{2+}$ by membrane pumps then reduces the $\mathrm{Ca}^{2+}$ concentration to lower levels. If the $\mathrm{IP}_{3}$ concentration is in the correct range, it can initiate a repeating cycle of release and uptake of $\mathrm{Ca}^{2+}$ from the ER, resulting in $\mathrm{Ca}^{2+}$ oscillations.

Our model here is based on a model of the $\mathrm{IP}_{3}$ receptor which corresponds to the binding diagram in Fig. 1, where $X$, $Y$, and $Z$ are the three possible states for the receptor, and the rates of interconversion between them are functions of the intracellular calcium concentration $c . X$ represents the fraction of $\mathrm{IP}_{3}$ receptors in the open state at any given time, $Y$ is the fraction of $\mathrm{IP}_{3}$ receptors in the shut state at any given time, and $Z$ is the fraction of $\mathrm{IP}_{3}$ receptors in the inactivated state at any given time. To simplify the model, we assume that interconversion between the open and shut states is rapid compared to the process of receptor inactivation and recovery from inactivation. This places an additional constraint on the variables, so that now model behavior is determined by just two variables: calcium concentration $(c)$, and the fraction of receptors in either the open or shut states. Letting $h$ equal this fraction (i.e., $h=X+Y$ ), the differential equation for $h$ is
TABLE I. Parameter values of the model.

\begin{tabular}{ll}
\hline \hline$k_{f}=28 \mu \mathrm{M} \mathrm{s}^{-1}$ & $J_{\text {leak }}=0.2 \mu \mathrm{M} \mathrm{s}^{-1}$ \\
$V_{p}=1.2 \mu \mathrm{M} \mathrm{s}^{-1}$ & $K_{p}=0.18 \mu \mathrm{M}$ \\
$R_{5}=1.6 \mu \mathrm{M}$ & $k_{2}=26.5 \mu \mathrm{M} \mathrm{s}^{-1}$ \\
$R_{3}=50 \mu \mathrm{M}$ & $k_{1}=44 \mu \mathrm{M} \mathrm{s}^{-1}$ \\
$r_{2}=100 \mathrm{~s}^{-1} \mu \mathrm{M}^{-1}$ & $r_{4}=20 \mathrm{~s}^{-1}$ \\
$R_{1}=6 \mu \mathrm{M}$ & $k_{3}=1.6 \mu \mathrm{M} \mathrm{s}^{-1}$ \\
\hline
\end{tabular}

$$
\frac{d h}{d t}=\phi_{3}(c)(1-h)-\frac{\phi_{1}(c) \phi_{2}(c) h p}{\phi_{1}(c) p+\phi_{-1}(c)} .
$$

The specific forms used for the rate functions are taken from Ref. 13, which in turn are based on a modification of the model of Ref. 12. For our rate functions, we choose

$$
\begin{aligned}
& \phi_{1}(c)=\frac{r_{2} c}{R_{1}+c}, \\
& \phi_{-1}(c)=\frac{k_{1}}{R_{3}+c}, \\
& \phi_{2}(c)=\frac{k_{2}+r_{4} c}{R_{3}+c}, \\
& \phi_{3}(c)=\frac{k_{3}}{R_{5}+c},
\end{aligned}
$$

where $p$ is the $\mathrm{IP}_{3}$ concentration, the main controlling parameter. We call this the two-state model. The $r \mathrm{~s}, R \mathrm{~s}$, and $k \mathrm{~s}$ are constants, with values given in Table I.

We incorporate the above model of the $\mathrm{IP}_{3}$ receptor into a model for intracellular $\mathrm{Ca}^{2+}$ dynamics by assuming that $\mathrm{Ca}^{2+}$ enters the cell either through the $\mathrm{IP}_{3}$ receptor $\left(J_{\text {receptor }}\right)$ or through a generic leak $\left(J_{\text {leak }}\right)$, and that $\mathrm{Ca}^{2+}$ leaves the cytoplasm only by being pumped out by $\mathrm{Ca}^{2+}$ ATPase pumps $\left(J_{\text {pump }}\right)$. Conservation of $\mathrm{Ca}^{2+}$ gives

$$
\frac{d c}{d t}=J_{\text {receptor }}-J_{\text {pump }}+J_{\text {leak }} .
$$

$J_{\text {leak }}$ is defined to be constant, $J_{\text {pump }}$ is modeled as a Hill equation with Hill coefficient of 2 , and $J_{\text {receptor }}$ is just a constant multiplied by the open probability of the $\mathrm{IP}_{3}$ receptor. Thus

$$
\begin{aligned}
& J_{\text {pump }}=\frac{V_{p} c^{2}}{K_{p}^{2}+c^{2}}, \\
& J_{\text {receptor }}=k_{f}\left(\frac{p h \phi_{1}(c)}{\phi_{1}(c) p+\phi_{-1}(c)}\right)^{4} .
\end{aligned}
$$

The open probability has the fourth power because the $\mathrm{IP}_{3}$ receptor is composed of four identical subunits and all of the subunits must be in the open state for the receptor to be open.

The major question which we want to address in this article is what happens when two of the above cellular models are coupled together by allowing intracellular $\mathrm{Ca}^{2+}$ to diffuse between them? In order to do this, we modified the differential equation for calcium slightly, so that it has the following form: 


$$
\frac{d c_{1}}{d t}=J_{\text {receptor }}-J_{\text {pump }}+J_{\text {leak }}+J_{\text {diffusion }} .
$$

Note that since there are now two cells, there are two calcium concentrations, $c_{1}$ and $c_{2}$, and two receptor variables, $h_{1}$ and $h_{2}$. We choose to model the diffusion as simply as possible and so we assume the linear form

$$
J_{\text {diffusion }}=D\left(c_{2}-c_{1}\right),
$$

where $D$ is a rate constant, with units of $1 /$ time, that acts as a measure of the permeability of the intercellular coupling. This is a similar formulation to that found in more detailed models of intercellular $\mathrm{Ca}^{2+}$ waves. ${ }^{3,14}$ The equations for $h_{1}$ and $h_{2}$ are unchanged from the original equation for $h$, with $c_{1}$ only appearing in the equation for $h_{1}$, and $c_{2}$ only appearing in the equation for $h_{2}$.

\section{A. Summary of the model equations}

$$
\begin{aligned}
\frac{d c_{1}}{d t}= & k_{f}\left(\frac{p h_{1} \phi_{1}\left(c_{1}\right)}{\phi_{1}\left(c_{1}\right) p+\phi_{-1}\left(c_{1}\right)}\right)^{4}-\frac{V_{p} c_{1}^{2}}{K_{p}^{2}+c_{1}^{2}} \\
& +J_{\text {leak }}+D\left(c_{2}-c_{1}\right), \\
\frac{d h_{1}}{d t}= & \phi_{3}\left(c_{1}\right)\left(1-h_{1}\right)-\frac{\phi_{1}\left(c_{1}\right) \phi_{2}\left(c_{1}\right) h_{1} p}{\phi_{1}\left(c_{1}\right) p+\phi_{-1}\left(c_{1}\right)}, \\
\frac{d c_{2}}{d t}= & k_{f}\left(\frac{p h_{2} \phi_{1}\left(c_{2}\right)}{\phi_{1}\left(c_{2}\right) p+\phi_{-1}\left(c_{2}\right)}\right)^{4}-\frac{V_{p} c_{2}^{2}}{K_{p}^{2}+c_{2}^{2}} \\
& +J_{\text {leak }}-D\left(c_{2}-c_{1}\right), \\
\frac{d h_{2}}{d t}= & \phi_{3}\left(c_{2}\right)\left(1-h_{2}\right)-\frac{\phi_{1}\left(c_{2}\right) \phi_{2}\left(c_{2}\right) h_{2} p}{\phi_{1}\left(c_{2}\right) p+\phi_{-1}\left(c_{2}\right)} .
\end{aligned}
$$

The parameter values used throughout the article are found in Table I. Although the use of different functions for the $\phi \mathrm{s}$ will result in quantitatively different results, it is not clear how the qualitative nature of the results will be affected. To determine this, one would have to study all the variants of the model in detail, something we have not yet done. Furthermore, there are many other models for intracellular $\mathrm{Ca}^{2+}$ dynamics, many of which show great qualitative similarity, but differ in the details. Hence, neither is it clear how our specific results here will apply to other models. However, there are indications that the overall results will remain unaffected, and we shall discuss this in more detail later.

Clearly, this is a very simple way to model the coupling of cellular oscillators, and will introduce a number of inaccuracies. However, we believe that it is necessary first to understand this simplest case before more realistic and complex scenarios are considered.

\section{BEHAVIOR OF TWO IDENTICAL COUPLED CELLS}

We examined the behavior of the coupled system numerically, using $\mathrm{AUTO}^{15}$ and xpp, as implemented in xppaut by B. Ermentrout (http://www.pitt.edu/phase). As a numerical check, all stable oscillatory solutions found by AUTO were also found by direct numerical solution with xpp.

Before beginning the analysis of the coupled system, it will be useful to summarize the long-term behavior of the

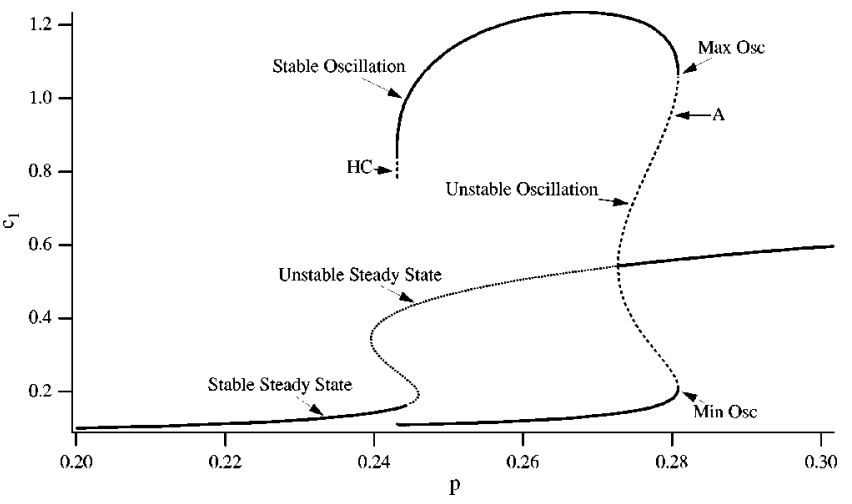

FIG. 2. Bifurcation diagram for large $D\left(D=1 \mathrm{~s}^{-1}\right)$. This is essentially identical to the diagram for $D=0$. Branch A is a branch of oscillatory solutions. In all the figures, a dotted line indicates instability, and a solid line indicates stability. HC denotes a homoclinic bifurcation. Here, and in all the figures, $c_{1}, c_{2}$, and $p$ are in units of $\mu \mathrm{M}$.

uncoupled cell (i.e., $D=0$ ). Figure 2 is a bifurcation diagram showing the steady states and oscillatory solutions as well as their stability, as $p$ (the $\mathrm{IP}_{3}$ concentration) is varied. For small $p$ there is a single stable steady state; then, as $p$ is increased, two unstable steady states appear in a saddle-node (SN) bifurcation. Then the stable fixed point becomes unstable in a Hopf bifurcation, and combines with the lower unstable steady state to disappear in another SN bifurcation. This leaves only the upper unstable fixed point as $p$ continues to increase. Finally, this fixed point gains stability in a Hopf bifurcation, and remains stable as $p$ continues upward. The upper Hopf bifurcation is subcritical, but the resultant branch of unstable periodic orbits turns around in a saddlenode of periodics bifurcation, giving rise to a branch of stable periodic orbits, which ends in a homoclinic bifurcation (HC). Note also that all long-term behavior of a single cell must show up as possible long-term behavior for our two identical coupled cells, since if $c_{1}=c_{2}$ and $h_{1}=h_{2}$ initially, they will be equal always, and no net diffusion of $\mathrm{Ca}^{2+}$ will take place. This is essentially the same as setting $D=0$, which just gets us back to the original, uncoupled model.

\section{A. Fixed point analysis}

The first question we analyzed was what happened to the number and stability of fixed points when the cells were coupled. The uncoupled cell had one, two, or three fixed points depending on the value of the parameter $p$ (see Fig. 2). We found that two coupled cells could have one, two, three, five, or nine fixed points, depending on the choices of $p$ and D.

For large $D(D>1)$, diffusion occurs so freely that the cells are very tightly coupled together, meaning that there are no fixed points where $c_{1} \neq c_{2}$. Of course, all double solutions (where $c_{1}=c_{2}$ in the long term) must be solutions for the uncoupled cell, and therefore, the steady states for the uncoupled cell are the same as for the coupled cells with large $D$ (see Fig. 2).

For smaller values of $D$, the diagram begins to get more complicated. When $D=0.1 \mathrm{~s}^{-1}$ there is an additional ring of unstable steady states which appears in the middle of the $\mathrm{S}$-shaped curve. As $D$ is decreased, the new ring of steady 


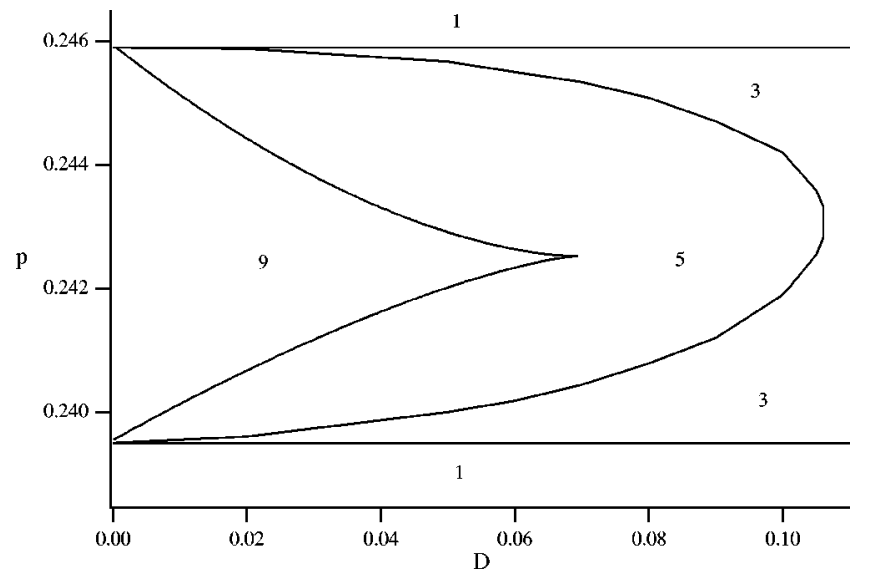

FIG. 3. Two-parameter steady-state bifurcation diagram indicating the number of steady states in each region of parameter space.

states elongates and begins to twist around and become more $\mathrm{S}$-shaped itself (computations not shown). There is never any region where there are seven steady states, since the folding happens at the same value of $p$ for both the upper and lower part of the ring. As $D$ is decreased still further, the ring becomes more and more $\mathrm{S}$-shaped, similar to the original $\mathrm{S}$ of the uncoupled cell, until, when $D=0$, the different branches of steady states exactly coincide. Figure 3 is a twoparameter bifurcation diagram showing the number of steady states in each region of parameter space.

\section{B. Oscillatory solutions}

Coupled identical cells also exhibit complex stable oscillatory behavior of several types. Figure 4 shows a series of bifurcation diagrams for gradually decreasing $D$. Branches of oscillatory solutions which are unstable for all values of $D$ have been omitted from the figures. For convenience, only oscillatory branches are labeled, and they are labeled alphabetically in the order in which they appear.

Recall that for high values of $D$, the coupled cells have an identical bifurcation diagram to an uncoupled cell (see Fig. 2). As $D$ is decreased, a pair of Hopf bifurcations appears (at around $D=0.5 \mathrm{~s}^{-1}$ ) and separate on the center branch of the steady-state curve, with an unstable arc of oscillatory solutions linking them (computations not shown).

As $D$ is decreased further, the Hopf points on branch B continue to slide further apart and the arc between them expands and bifurcates into at least two different branches of periodic orbits. However, since these bifurcations all give rise to unstable orbits, we do not consider them in detail. Instead, we skip straight to $D=0.07$ as shown in Fig. 4(a). At this value of $D$, branch $\mathrm{B}$ has given rise to unstable branches $\mathrm{C}$ and $\mathrm{D}$, and additional branches $\mathrm{E}$ and $\mathrm{F}$ have appeared on branch A. Branches $\mathrm{F}$ and $\mathrm{E}$ have stable portions. As $D$ is decreased still further [Fig. 4(b)], branch C becomes very convoluted (although it remains unstable), and the stable parts of branches $\mathrm{E}$ and $\mathrm{F}$ grow longer. Branch $\mathrm{E}$ also begins to extend to the left as branch $\mathrm{D}$ begins to bend to meet it. Likewise, branch $\mathrm{F}$ begins to extend leftward as branch $\mathrm{C}$ begins to bend to meet it. The branches finally contact one another and reorganize; the key transition is from
Fig. 4(b) to Fig. 4(c). In Fig. 4(c) branches A and B remain but there are a number of new branches. Branches $\mathrm{G}$ and $\mathrm{H}$ are formed out of branches D and E; G by the lower parts of $\mathrm{D}$ and $\mathrm{E}$, and $\mathrm{H}$ by the upper parts of $\mathrm{D}$ and $\mathrm{E}$. Branch $\mathrm{J}$ is formed from the lower part of branch $\mathrm{F}$ and the upper part of branch $\mathrm{C}$, and branch I was born out of the upper part of branch $\mathrm{F}$ and the lower part of branch $\mathrm{C}$.

As $D$ is decreased still further, branches $\mathrm{H}$ and I become smaller and less pronounced, while branch $\mathrm{B}$ and branch $\mathrm{J}$ approach branch A. Branch $\mathrm{G}$ and branch $\mathrm{J}$ continue to have stable portions, and a small stable portion appears on branches $\mathrm{I}$ and $\mathrm{H}$ right where they meet, and where they cross branch B. At very small values of $D$ [see Fig. 4(d)], a small portion of branch B becomes stable. Ultimately, at $D$ $=0$, branches $\mathrm{B}, \mathrm{H}, \mathrm{I}$, and $\mathrm{J}$ all melt into branch $\mathrm{A}$, and branch $\mathrm{G}$ melts into the steady-state branch. This brings us back to a diagram identical to Fig. 2.

\section{Synchronized oscillations}

The coupled cells exhibit stable, synchronized, in-phase oscillations at all values of $D$, and only along branch A. As noted above, this was necessarily the case, since coupled cells with identical initial conditions behave as uncoupled cells. However, it does not necessarily have to be the case that the stability of the solution is the same as in the single cell. In fact, for intermediate values of $D$, a small portion of branch A which is stable in the uncoupled cell becomes unstable in the coupled cells. This can be seen in the difference between Fig. 2 and Fig. 4(d). In Fig. 2, branch A becomes stable right at its limit point, whereas in Fig. 4(d), branch A clearly does not become stable until well above its limit point. Hence the uncoupled stability is not a good predictor of coupled stability. This is discussed further below (see Fig. $6)$.

\section{Different-amplitude nearly in-phase oscillations}

Figure 5(a) shows a few cycles of this type of behavior. This solution has large amplitude oscillations for one cell, and small amplitude oscillations for the other, with the small amplitude cell leading the larger one by about 15 degrees. Remember that since the cells are identical, either cell can be the one with the large amplitude oscillation. This type of behavior is present in Fig. 4. In Figs. 4(a) and 4(b), the large amplitude oscillation is found on the stable part of branch $\mathrm{F}$ and the small amplitude oscillation is found on the stable part of branch E. In Figs. 4(c) and 4(d), the large amplitude oscillation is found on the stable part of branch $\mathbf{J}$ and the small amplitude oscillation is found on the stable part of branch $\mathrm{G}$.

\section{Same-amplitude antiphase oscillations}

For a small range of $p$ and $D$ values, the coupled cells also exhibited same amplitude antiphase oscillations [see Fig. 5(b)]. This behavior corresponds to the stable part of branch B in Fig. 4(d). 
$\mathbf{A}$

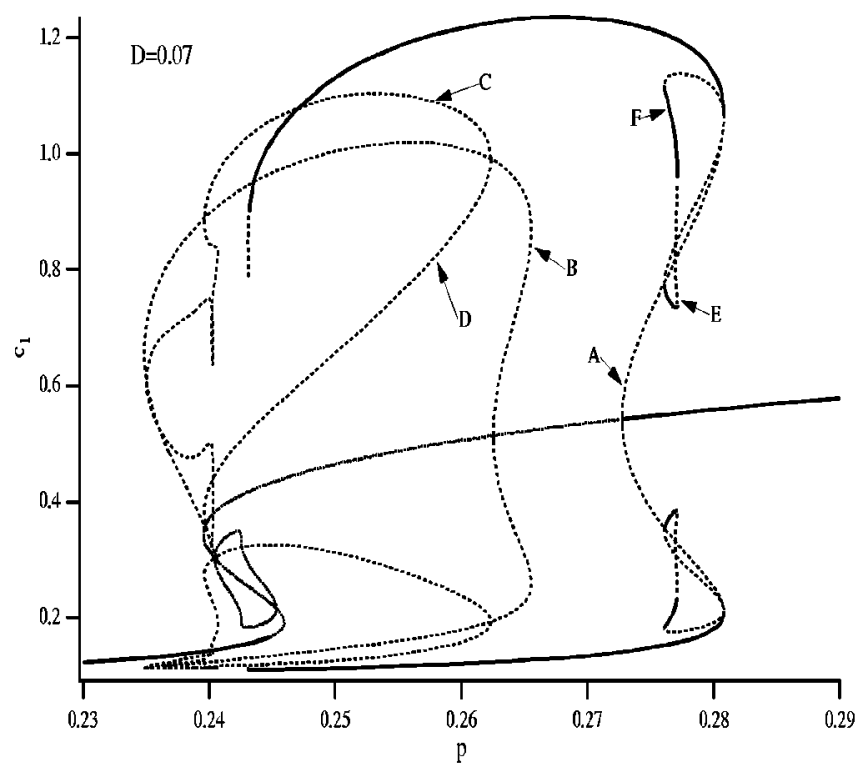

C

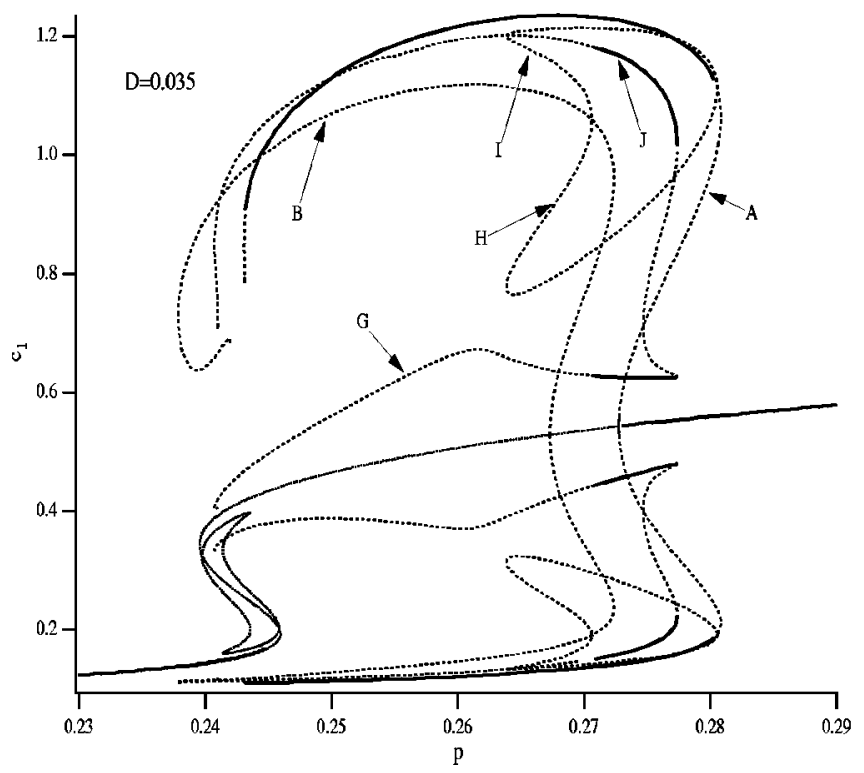

B

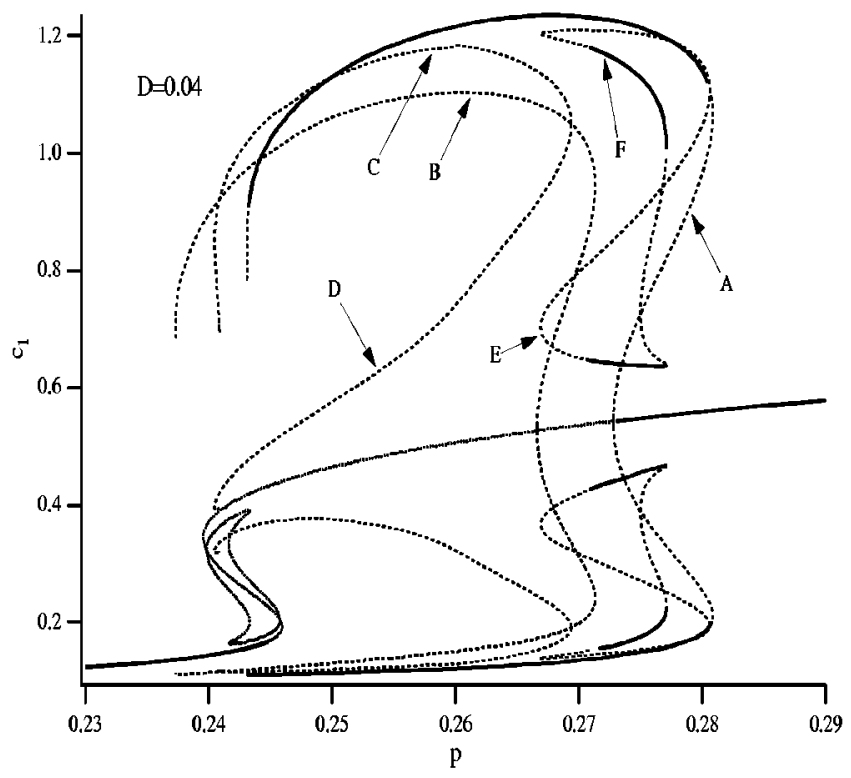

D

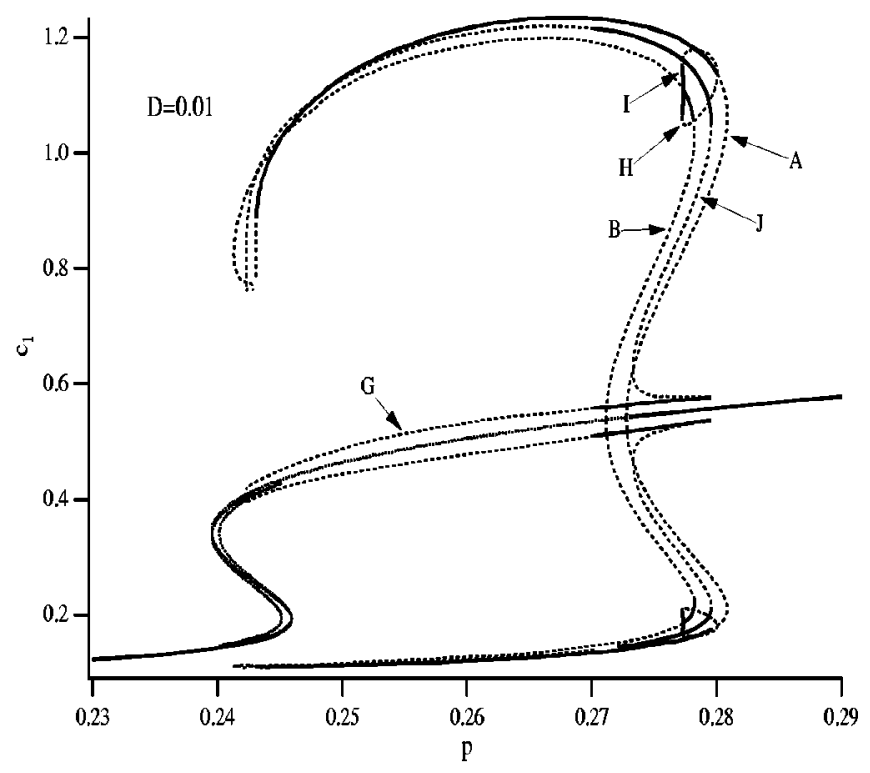

FIG. 4. Bifurcation diagrams for $D=0.07,0.04,0.035$, and $0.01 \mathrm{~s}^{-1}$.

\section{Different-amplitude antiphase oscillations}

The stable branches splitting off the same amplitude antiphase oscillations [branches H and I in Fig. 4(d)] are slight deformations of them, where the oscillations are still antiphase, but one of the cells oscillates slightly higher, and the other oscillates slightly lower, so that they have different amplitudes. The higher amplitude oscillation is on branch I, the lower on branch $\mathrm{H}$.

\section{A two-parameter diagram}

In Fig. 6 we summarize the important behaviors in the previous figures, by plotting the positions of the important bifurcations as $D$ and $p$ vary. LP denotes the limit point on branch A (i.e., the saddle node of periodic bifurcation), and HB denotes the position of the Hopf bifurcation at which branch A first appears.

Note that there are four curves (labeled 1 to 4 ), each with an upper and a lower branch.

\section{Curve 1}

Curve 1 shows the position of the two bifurcation points at which branches $\mathrm{F}$ and $\mathrm{E}$ first appear (at $D \approx 0.09$ ). As $D$ decreases, the loop of unstable steady states formed by branches $\mathrm{F}$ and $\mathrm{E}$ expands, with the upper bifurcation point moving up, the lower one moving down. When $D \approx 0.07$, the upper bifurcation point coincides with the limit point [see 
$\mathbf{A}$

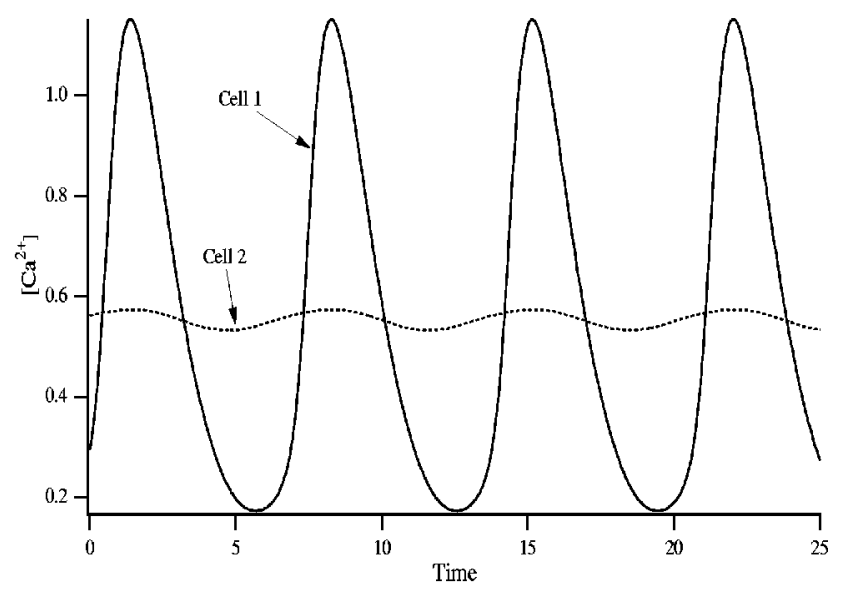

B

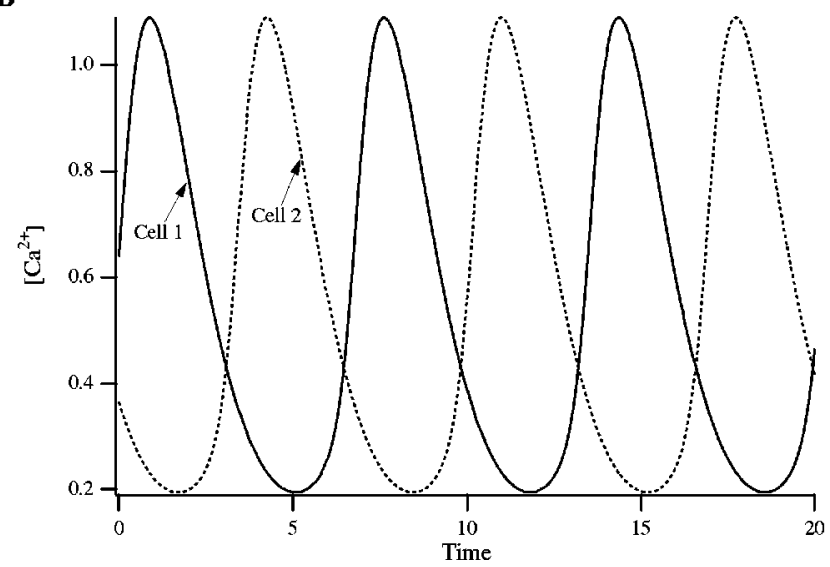

FIG. 5. (a) Calcium oscillations from branch J of Fig. 4(d), $D=0.01 \mathrm{~s}^{-1}$, $p=0.2778 \mathrm{mM}$. (b) Calcium oscillations from branch B of Fig. 4(d), $D$ $=0.01 \mathrm{~s}^{-1}, p=0.2778 \mathrm{mM}$.

Fig. 4(a)], but then, as $D$ decreases further, the upper bifurcation point continues to move around branch $\mathrm{A}$. This can be seen clearly in Fig. 6, as the upper branch of curve 1 initially moves up (as $D$ decreases), merges briefly with the LP line, but then moves down again. As $D \rightarrow 0$, the upper branch of

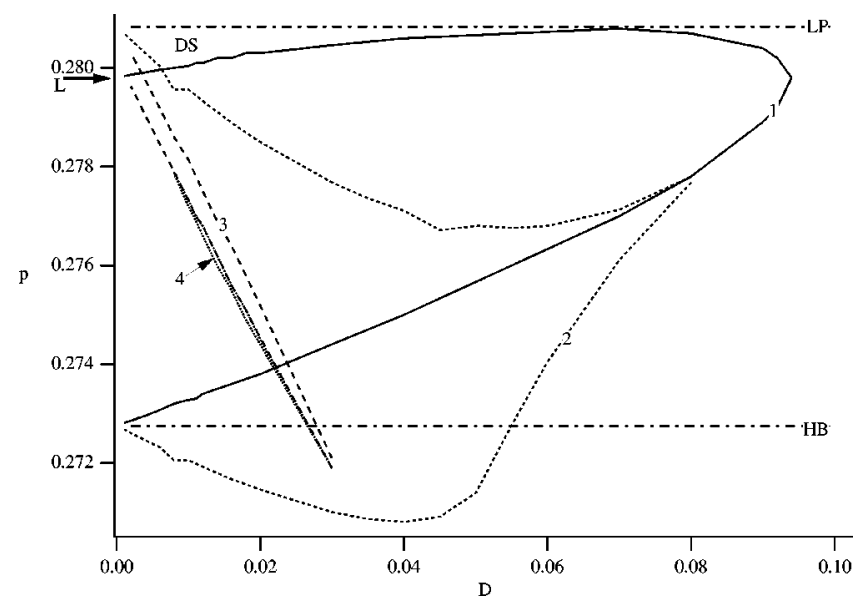

FIG. 6. Two-parameter bifurcation diagram showing the positions of the major bifurcations as functions of $p$ and $D$. curve 1 merges with the lower branch of curve 3 (at the point labeled L in Fig. 6), while the lower branch of curve 1 merges with the Hopf bifurcation, HB.

Consider the region marked DS in Fig. 6, i.e., the region to the left of $D \approx 0.07$, and for $p$ between LP and the upper branch of curve 1 . In region DS, same-amplitude, in-phase oscillations are unstable. In the uncoupled case sameamplitude, in-phase oscillations are always stable when $p$ is less than LP, and thus the region DS corresponds to values for $D$ and $p$ such that the coupling has destabilized an oscillatory solution.

\section{Curve 2}

Curve 2 shows the positions of the bifurcations that denote the upper and lower limits of the stable portion of oscillatory branch F [and E; see Figs. 4(a) and 4(b)]. As D decreases, the upper branch of curve 2 merges with LP, while the lower branch merges with HB. Thus, for any given value of $D$, if $p$ lies between the upper and lower branches of curve 2 , then stable different-amplitude in-phase oscillations exist.

\section{Curve 3}

Curve 3 shows the positions of the bifurcations that denote the upper and lower limits of the stable portion of oscillatory branch B [see Fig. 4(d)]. Thus, for any given value of $D$, if $p$ lies between the upper and lower branches of curve 3 , then stable same-amplitude anti-phase oscillations exist. Note that, as $D \rightarrow 0$, the upper branch of curve 3 tends to LP, while the lower branch merges with the upper branch of curve 1 , at point $\mathrm{L}$.

\section{Curve 4}

Curve 4 shows the positions of the bifurcations that denote the upper and lower limits of the stable portion of oscillatory branch $\mathrm{H}$ [see Fig. 4(b)]. Thus, for any given value of $D$, if $p$ lies between the upper and lower branches of curve 4 , then stable different-amplitude anti-phase oscillations exist. Curve 4 exists for only a very narrow range of values for $p$ and $D$.

The most interesting result to appear from this twoparameter bifurcation diagram is the behavior of the model for infinitesimally small coupling. When $D=0$ we have stable same-amplitude oscillations for all $p$ less than LP (at least until they disappear in the homoclinic bifurcation at much lower values of $p$, as shown in Fig. 2). Because of the absence of coupling, these oscillations can have an arbitrary phase difference. However, as soon as $D$ increases from 0 , this structure breaks down and only a selection of possible periodic orbits remains stable. If $p$ is less than $\mathrm{HB}$, only same-amplitude in-phase orbits remain stable. If $p$ is between $\mathrm{HB}$ and $\mathrm{L}$, then same-amplitude in-phase orbits are still stable, but different-amplitude nearly-in-phase oscillations are also stable. Finally, if $p$ is between L and LP the sameamplitude in-phase orbits have lost stability, while differentamplitude in-phase oscillations, or same-amplitude antiphase oscillations are now stable. Which one is finally selected will depend on the initial conditions. 

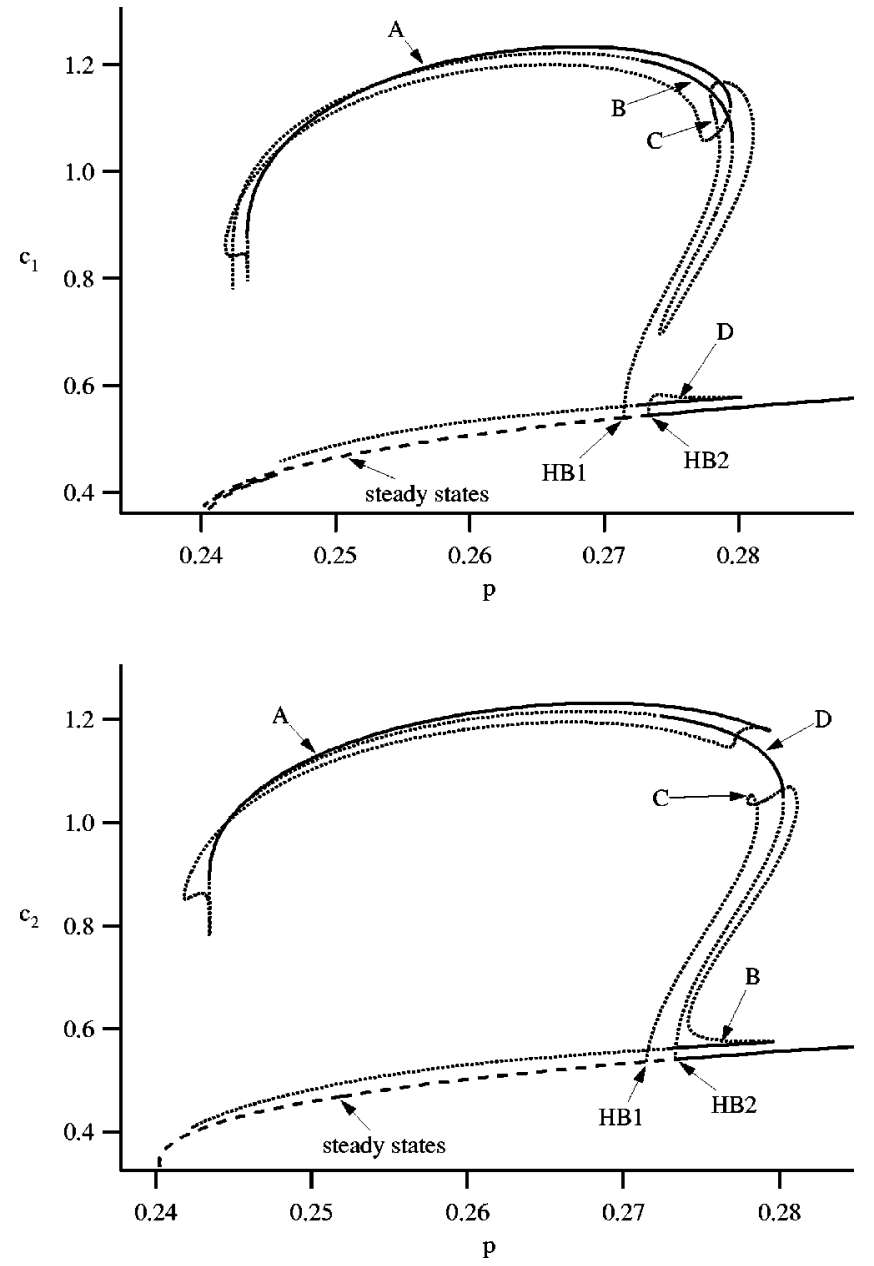

FIG. 7. Bifurcation diagrams for two similar but nonidentical cells $\left(k_{f 1}\right.$ $\left.=28 \mu \mathrm{M} \mathrm{s}^{-1}, k_{f 2}=27.9 \mu \mathrm{M} \mathrm{s}^{-1}, D=0.01 \mathrm{~s}^{-1}\right)$. The upper panel plots $c_{1}$ against $p$, the lower panel plots $c_{2}$ against $p$. Unstable branches of steady states are denoted by dashed lines. Unstable branches of periodic orbits are denoted by dotted lines. HB1 and HB2 denote two Hopf bifurcations. The four branches of stable periodic orbits are labeled A, B, C, and D.

\section{COUPLED NONIDENTICAL CELLS}

In reality one could never observe two coupled identical cells, and thus it is of much greater physiological importance to understand the behavior of coupled nonidentical cells. It turns out that if the two cells are almost identical, all of the above behaviors persist, and that as the differences become greater more complex behaviors appear.

To model nonidentical cells we chose to vary the parameter $k_{f}$. This corresponds to cells with different densities of $\mathrm{IP}_{3}$ receptors, a situation that is well known to occur experimentally. Thus, for cell 1 we keep $k_{f 1}=28 \mu \mathrm{M} \mathrm{s}^{-1}$, while for cell 2 we let $k_{f 2}$ take a range of values, from 27.9 to $24 \mu \mathrm{M} \mathrm{s}^{-1}$.

\section{A. Almost identical cells}

First we consider the case $k_{f 2}=27.9 \mu \mathrm{M} \mathrm{s}^{-1}$, i.e., cell 2 is almost identical to cell 1. In Fig. 7 we plot bifurcation diagrams for the case $D=0.01 \mathrm{~s}^{-1} ; c_{1}$ against $p$ in the upper panel, and $c_{2}$ against $p$ in the lower. These diagrams should be compared to that in Fig. 4(d), which is the corresponding diagram for the case of identical cells.
Just as in the case of identical cells, there are two Hopf bifurcations (HB1 and HB2) along the curve of steady states. Each of these Hopf bifurcations gives rise to a branch of periodic orbits. The branch arising from HB1 forms a large loop, and has two stable portions, labeled $\mathrm{B}$ and $\mathrm{C}$, respectively. The branch arising from HB2 gives rise to the stable periodic orbits labeled D. Note that branch $\mathrm{C}$ of stable periodic orbits is small, and not easy to see in the figure. However, its existence is easily confirmed by direct numerical solution.

Branch B of periodic orbits corresponds to differentamplitude in-phase oscillations, with $c_{1}$ oscillating with a large amplitude, and $c_{2}$ oscillating with a small amplitude. Similarly, branch D corresponds to different-amplitude inphase oscillations, with $c_{1}$ oscillating with a small amplitude, and $c_{2}$ oscillating with a large amplitude. These periodic solutions are analogous to the stable portions of branches $\mathbf{J}$ and $\mathrm{G}$ in Fig. 4(d).

Branch C corresponds to same-amplitude anti-phase oscillations, and is analogous to branches H and I in Fig. 4(d).

Although the same kinds of stable oscillations appear in both Fig. 4(d) and Fig. 7 (i.e., when the cells are either identical or only slightly different), it is interesting to note that the bifurcation diagrams have qualitatively different structures. First, when the cells are not identical, branch A (corresponding to in-phase same-amplitude oscillations) forms a closed isolated loop that is separated from the other branches of the diagram; such a loop is called an isola. Furthermore, in Fig. 7 a second loop is formed by the branch of orbits arising at HB1. One can see that, by joining the loop and the isola, one can reconstruct the structure of the bifurcation diagram in Fig. 4(d). Hence, by making the cells nonidentical, the structure of the bifurcation diagram has been "pulled apart," resulting in an isola and a separate loop.

\section{B. Less similar cells}

When we decrease $k_{f 2}$ to $24.0 \mu \mathrm{M} \mathrm{s}^{-1}$ most of the same overall behaviors persist, but more complex behaviors also appear (Fig. 8). It is useful to note that when $p=0.27 \mu \mathrm{M}$ the ratio of the periods of the two coupled cells is now 1.34. Although this ratio varies with $p$, it is still a useful measure of the extent of similarity between the cells.

When $k_{f 2}=24 \mu \mathrm{M} \mathrm{s}^{-1}$ the most obvious type of oscillation that has been lost is the same-amplitude anti-phase oscillation (on branch C in Fig. 7); this disappears by the time $k_{f 2}$ has been decreased to $27.0 \mu \mathrm{M} \mathrm{s}^{-1}$. When $k_{f 2}$ $=24 \mu \mathrm{M} \mathrm{s}^{-1}$ there are three branches of stable periodic orbits, labeled A, B, and C. Branch C corresponds to periodic orbits in which cell 1 is oscillating with a large amplitude, and cell 2 with a small amplitude. In other words, during each oscillation of cell 1 , a small amount of $\mathrm{Ca}^{2+}$ "leaks through" to cell 2, thus driving a small-amplitude oscillation in the coupled cell. (Because $k_{f 2}=24 \mu \mathrm{M} \mathrm{s}^{-1}$, an isolated cell 2 is not capable of generating spontaneous oscillations at such low values for $p$.) Branch A is just the converse of this; spontaneous oscillations in cell 2 leak through to drive smallamplitude oscillations in cell 1 .

Branch B is more interesting, as it corresponds to stable 

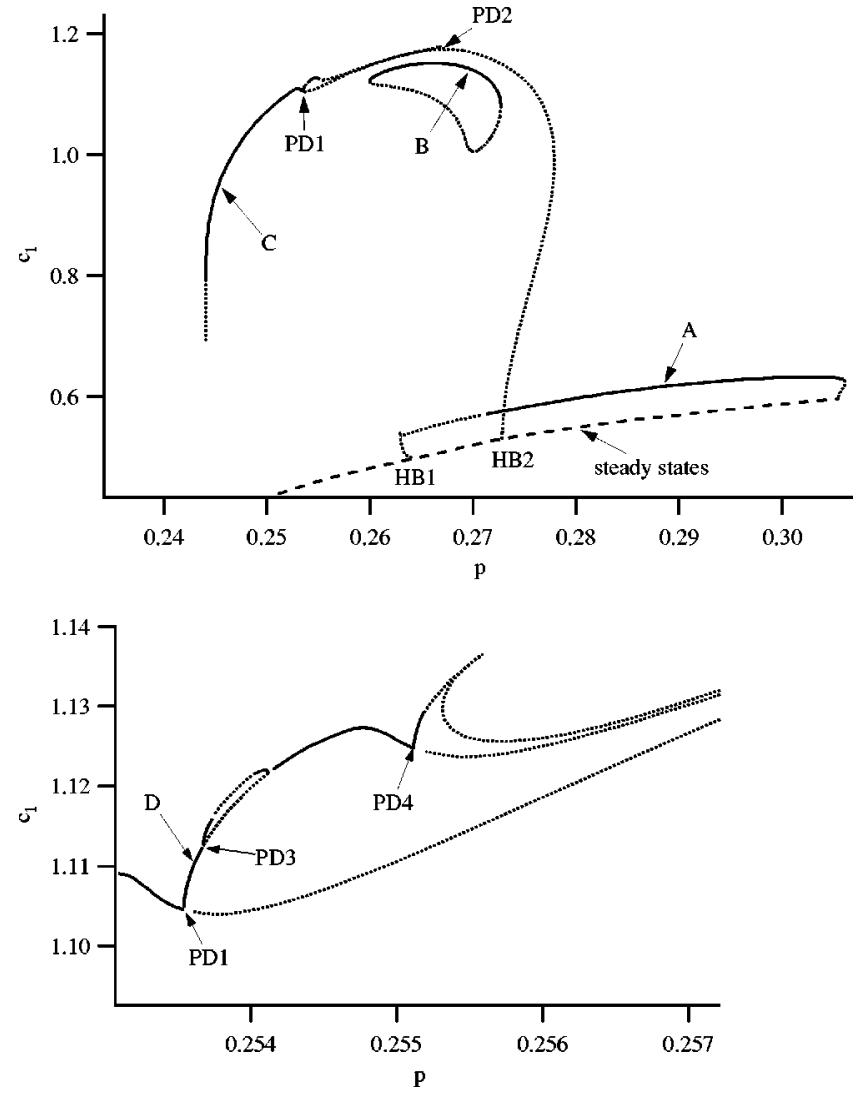

FIG. 8. Bifurcation diagrams for two nonidentical cells $\left(k_{f 1}=28 \mu \mathrm{M} \mathrm{s}^{-1}\right.$, $\left.k_{f 2}=24 \mu \mathrm{M} \mathrm{s}^{-1}, D=0.05 \mathrm{~s}^{-1}\right)$. The upper panel plots $c_{1}$ against $p$, the lower panel shows a detail of the bifurcation diagram around PD1. HB1 and HB2 denote two Hopf bifurcations. PD denotes a period doubling bifurcation. Unstable branches of steady states are denoted by dashed lines. Unstable branches of periodic orbits are denoted by dotted lines.

phase-locked oscillations, in which each cell oscillates with large amplitude. The isola of Fig. 7 has now shrunk considerably, resulting in a smaller range of values for $p$ that give rise to phase-locked similar-amplitude oscillations.

On branch $\mathrm{C}$ there is also a cascade of period-doubling bifurcations, at which more complex solutions arise. The lower panel of Fig. 8 shows a blow-up of the bifurcation structure around the lower period-doubling bifurcation. Branch D corresponds to period-2 orbits that wind twice around the steady-state before repeating, and it itself undergoes further period-doubling bifurcations to give yet more complicated orbits. We did not trace the bifurcation structure past the first two period-doubling bifurcations. A selection of multiply-periodic orbits is shown in Fig. 9, for increasing values of $p$. As $p$ is increased, the phase-locked differentamplitude periodic orbits bifurcate to period-2 orbits (i.e., on branch D). [See Fig. 9(a)]. Further increases in $p$ lead to period-3 and period-4 orbits [Figs. 9(b) and 9(c)]. When $p$ is increased still further, the orbit falls on to the isola of phaselocked similar-amplitude orbits.

If we fix $p=0.27 \mu \mathrm{M}$ and vary $D$, a similar picture emerges (computations not shown). As $D$ increases to 0.07, the isola (branch B of Fig. 8) merges with the branch of unstable periodic orbits, and expands in size. As $D$ decreases, trajectories lose phase-locking, and the phase of cell 2 starts a

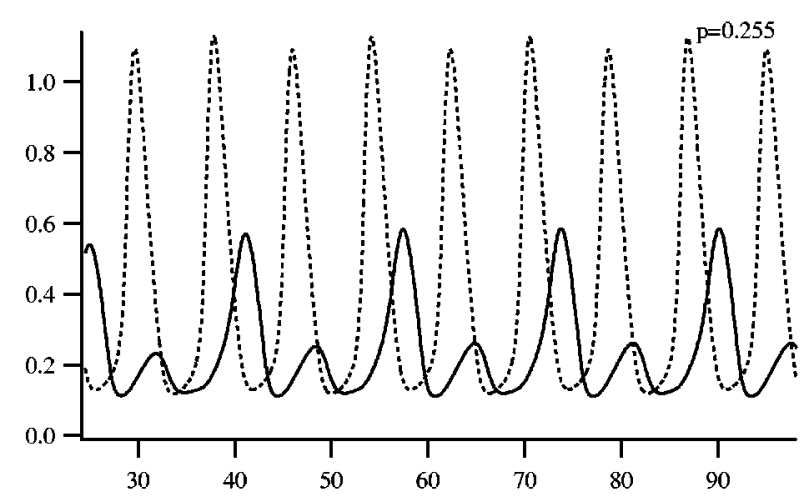

b

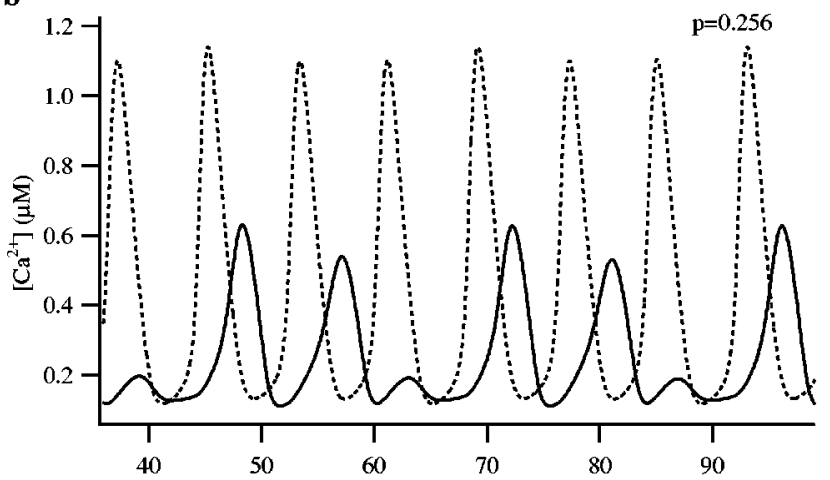

c

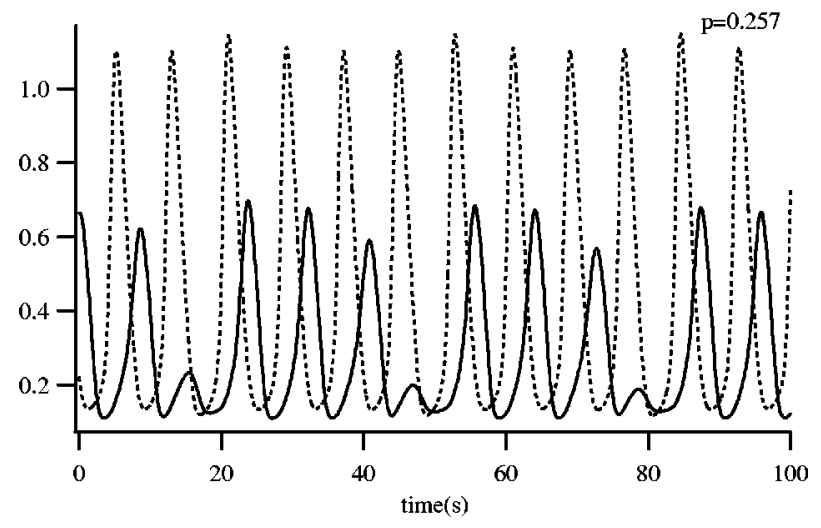

FIG. 9. Plots of $c_{1}$ and $c_{2}$ as functions of time, for increasing values of $p$. Here, $D=0.05 \mathrm{~s}^{-1} \cdot c_{1}$ is shown as a dotted curve, $c_{2}$ as a smooth curve.

to drift, as is commonly seen in other coupled oscillatory systems. At $D=0$, of course, the phases of the two cells are independent.

\section{DISCUSSION}

For a small range of parameter values, coupled identical cells can exhibit a wide variety of behaviors. For example, when $p=0.2778 \mu \mathrm{M}$ and $D=0.01 \mathrm{~s}^{-1}$, each cell can settle into a stable steady state, oscillate in synchrony with the other cell, oscillate in antiphase with the other cell, undergo large oscillations in intracellular $\mathrm{Ca}^{2+}$ while the other cell undergoes small oscillations in intracellular $\mathrm{Ca}^{2+}$, or undergo small oscillations in $\mathrm{Ca}^{2+}$ while the other cell undergoes large oscillations in $\mathrm{Ca}^{2+}$, and all of these behaviors are stable. For large $D$, the cells are locked in step and behave as one uncoupled cell. As $D$ is decreased, more complex behaviors gradually become possible, first different amplitude in- 
phase oscillations, then slightly different amplitude antiphase oscillations, and finally antiphase oscillations. These behaviors only disappear as $D$ reaches zero, uncoupling the cells.

When the cells are similar, but not identical, other complex behaviors, such as multiply-periodic orbits, appear. However, cellular heterogeneity quickly destroys the stability of the antiphase solutions; at realistic levels of intercellular heterogeneity the only physiologically significant solutions are the in-phase similar-amplitude solutions, the inphase different-amplitude solutions, or a variety of multiplyperiodic solutions. Note that there is a wide range of values for $p$ such that the only stable periodic solutions are multiply periodic (i.e., between branches B and C of Fig. 8) and thus we would expect to observe such solutions experimentally. We performed large numbers of simulations using arbitrary values of $p$ in this range; although the solutions often looked chaotic, we made no attempt to distinguish between true chaos or just a multiply-periodic solution of high period.

Our model is highly simplified, having no spatial dependence within each cell. It is not yet clear how such spatial dependencies will affect the results. However, it is well known that calcium oscillations often do not occur in a spatially homogeneous manner, but instead take the form of oscillatory intracellular waves. The theory of periodic waves in an oscillatory medium has been studied by a number of authors, most notably Ref. 16, who showed that the gradient of the phase in such a system obeys Burger's equation. Hence, a more physiologically realistic model of two coupled calcium oscillators would involve the coupling of two regions, each with underlying oscillatory kinetics, so as to obtain two coupled Burger's equations for the phase gradient. We leave discussion of this more detailed model for future work, noting only that the present results are a necessary preliminary to this more detailed study. Oscillatory waves also occur within single cells, particularly ones as large as the Xenopus oocyte, ${ }^{17}$ and these waves have been modeled in detail. ${ }^{18-20}$ It appears that in some circumstances the phase of these waves obeys Burger's equation, as predicted by Neu, and that the oscillatory kinetics can give rise to unstable spiral waves and spatio-temporal chaos. However, our understanding of these waves, although somewhat more advanced, is still incomplete. The complexity caused by the intercellular boundaries makes the intercellular wave problem very much more difficult.

To date there have been two major studies of coupled calcium oscillators and their relationship to periodic intercellular calcium waves, both in hepatocytes. ${ }^{7,8}$ Both of these studies use a cellular model very similar to the one used here, relying upon sequential activation and inactivation of the $\mathrm{IP}_{3}$ receptor by $\mathrm{Ca}^{2+}$ to generate $\mathrm{Ca}^{2+}$ oscillations, although the precise details differ. Dupont et al. ${ }^{8}$ showed numerically that if one assumes a gradient of agonist receptor density, and then couples a line of (otherwise identical) cells by the diffusion of either $\mathrm{Ca}^{2+}$ or $\mathrm{IP}_{3}$ through gap junctions, one can obtain periodic intercellular waves similar to those seen experimentally. By comparing their theoretical results to their experimental data they predicted that diffusion of $\mathrm{IP}_{3}$ through gap junctions was a more plausible mechanism for the coordination of the intercellular wave. This is a different mechanism than that used in this article (as we assume that it is $\mathrm{Ca}^{2+}$ that moves between cells). If $\mathrm{IP}_{3}$ is assumed to be the diffusing messenger, then the oscillations in each cell are effectively uncoupled (as $\mathrm{IP}_{3}$ plays no dynamic role in the oscillatory mechanism). Thus, in the model of Dupont et al., each cellular oscillator has no direct effect upon its neighbors, and the coupling arises from the equilibration of $\mathrm{IP}_{3}$ over multiple cells. The study of Höfer ${ }^{7}$ differs from that of Dupont et al. in that he assumes that the cells are coupled by the diffusion of $\mathrm{Ca}^{2+}$ through gap junctions, and he assumes greater intercellular heterogeneity. Höfer concludes that coupling by the diffusion of $\mathrm{Ca}^{2+}$ can indeed synchronize heterogeneous cells, and studies the types of oscillations that can occur as the coupling strength changes. His conclusion that stable asynchronous solutions are destroyed by small heterogeneities is consistent with our results. Although stable asynchronous solutions still exist when $k_{f 2}=27.9 \mu \mathrm{M} \mathrm{s}^{-1}$ (branch $\mathrm{C}$ in Fig. 7), they seem to have disappeared by $k_{f 2}$ $=27 \mu \mathrm{M} \mathrm{s}^{-1}$. In other respects also, our results are consistent with those of Höfer. For instance, Höfer shows the existence of phase locking, harmonic locking, and phase drifting, all of which we observe also. As the intercellular coupling is decreased, or as intercellular heterogeneity is increased, phase-locked solutions lose synchrony, as we and Höfer have found. These similarities are to be expected, as coupled oscillators tend to have similar generic behaviors, irrespective of the exact model used to model the individual oscillators. However, Höfer's conclusion that it is $\mathrm{Ca}^{2+}$, not $\mathrm{IP}_{3}$, that synchronizes periodic intercellular waves in hepatocytes differs from the results of Dupont et al. One can only conclude that we still do not know the mechanisms underlying such periodic intercellular waves; only further work can resolve these differences.

Lines of coupled oscillators with a phase gradient have been studied, among others, by Ermentrout and Kopell, ${ }^{21}$ who found that although phase-locked waves do not exist in general, the phases can be locked in an average sense, a phenomenon called "phase trapping." However, they used much simpler models than the ones discussed here, and how well their results carry over to more complex models is not clear. Presumably, many qualitative features will remain unchanged. Second, their results relied on the existence of a frequency gradient along the line of coupled cells. Although Tordjmann et al. ${ }^{6}$ have postulated exactly such a frequency gradient in hepatocytes (as used in the modeling work of Ref. 8), there is no evidence for such a gradient in a ring of pancreatic acinar cells. Finally, Ermentrout and Kopell assumed that each cell was a point oscillator, with no spatial structure, an assumption that is not true for coupled calcium oscillators.

The theory of coupled oscillators has played a major role in the study of many biological systems, particularly the heart and the gastrointestinal tract (see Refs. 22 and 23 for discussion and further references), or on a more macroscopic level, circadian rhythms. All the qualitative behavior described in this article has been seen previously in other systems. For instance, in-phase and antiphase solutions are known for coupled equations of Hodgkin-Huxley or FitzHugh-Nagumo type, ${ }^{24,25}$ and for coupled neural oscilla- 
tors acting as model of the circadian pacemaker. ${ }^{26}$ Models of coupled bursters such as the pancreatic $\beta$-cell have also been observed to sustain oscillations of different amplitudes, as well as in-phase and antiphase oscillations. ${ }^{27}$ That large $D$ leads to synchronization has been shown by Ref. 28, while the coexistence of multiple stable oscillations of different types has been shown in an enzyme system. ${ }^{29}$ A more general analysis of linearly coupled oscillators has been performed in Ref. 30, and Ref. 31 performed a detailed analytical and numerical study of coupled systems that are close to a supercritical Hopf bifurcation. However, none of these studies answers the specific question that we ask here, that of how intracellular calcium oscillators might be expected to behave when coupled. To answer this question, and to have an answer mean something physiologically, it is necessary to study more specific models in greater detail.

\section{ACKNOWLEDGMENTS}

This work was supported by NSF Grant No. DMS 9706565 and NIH Grant No. R01GM56126. Bindshadler was supported by the NSF Research Experience for Undergraduates Program.

${ }^{1}$ M. J. Sanderson, A. C. Charles, S. Boitano, and E. R. Dirksen, “Mechanisms and function of intercellular calcium signaling,', Molecular and Cellular Endocrinology 98, 173-187 (1994).

${ }^{2}$ A. P. Thomas, G. S. J. Bird, G. Hajnóczky, L. D. Robb-Gaspers, and J. W. J. Putney, "Spatial and temporal aspects of cellular calcium signaling,", FASEB J. 10, 1505-1517 (1996).

${ }^{3}$ J. Sneyd, B. Wetton, A. C. Charles, and M. J. Sanderson, “Intercellular calcium waves mediated by diffusion of inositol trisphosphate: A twodimensional model,' Am. J. Physiol. 268, C1537-C1545 (1995a).

${ }^{4}$ L. D. Robb-Gaspers and A. P. Thomas, "Coordination of $\mathrm{Ca}^{2+}$ signaling by intercellular propagation of $\mathrm{Ca}^{2+}$ waves in the intact liver,' J. Biol. Chem. 270, 8102-8107 (1995).

${ }^{5}$ T. Tordjmann, B. Berthon, M. Claret, and L. Combettes, "Coordinated intercellular calcium waves induced by noradrenaline in rat hepatocytes: dual control by gap junction permeability and agonist,' EMBO J. 16, 5398-5407 (1997).

${ }^{6}$ T. Tordjmann, B. Berthon, E. Jacquemin, C. Clair, N. Stelly, G. Guillon, M. Claret, and L. Combettes, "Receptor-oriented intercellular calcium waves evoked by vasopressin in rat hepatocytes,' EMBO J. 17, 46954703 (1998).

${ }^{7}$ T. Höfer, " Model of intercellular calcium oscillations in hepatocytes: synchronization of heterogeneous cells," Biophys. J. 77, 1244-1256 (1999).

${ }^{8}$ G. Dupont, T. Tordjmann, C. Clair, S. Swillens, M. Claret, and L. Combettes, "Mechanism of receptor-oriented intercellular calcium wave propagation in hepatocytes,'” FASEB J. 14, 279-289 (2000).

${ }^{9}$ B. Zimmermann and B. Walz, 'Serotonin-induced intercellular calcium waves in salivary glands of the blowfly Calliphora erythrocephala," J. Physiol. 500, 17-28 (1997).
${ }^{10}$ M. Wilkins and J. Sneyd, "Intercellular spiral waves of calcium," J. Theor. Biol. 19, 299-308 (1998).

${ }^{11}$ D. I. Yule, E. Stuenkel, and J. A. Williams, "Intercellular calcium waves in rat pancreatic acini: mechanism of transmission,' Am. J. Physiol. 271, C1285-C1294 (1996).

${ }^{12}$ A. P. LeBeau, D. I. Yule, G. E. Groblewski, and J. Sneyd, "Agonistdependent phosphorylation of the inositol 1,4,5-trisphosphate receptor: a possible mechanism for agonist-specific calcium oscillations in pancreatic acinar cells,' J. Gen. Physiol. 113, 851-871 (1999).

${ }^{13}$ J. Sneyd, A. LeBeau, and D. I. Yule, "Traveling waves of calcium in pancreatic acinar cells: model construction and bifurcation analysis," Physica D (in press) (2000).

${ }^{14}$ J. Sneyd, J. Keizer, and M. J. Sanderson, "Mechanisms of calcium oscillations and waves: a quantitative analysis,' FASEB J. 9, 1463-1472 (1995b).

${ }^{15}$ E. Doedel, "Software for continuation and bifurcation problems in ordinary differential equations,' California Institute of Technology (1986).

${ }^{16}$ J. C. Neu, "Chemical waves and the diffusive coupling of limit cycle oscillators,' SIAM (Soc. Ind. Appl. Math.) J. Appl. Math. 36, 509-515 (1979).

${ }^{17}$ J. Lechleiter, S. Girard, E. Peralta, and D. Clapham, "Spiral calcium wave propagation and annihilation in Xenopus laevis oocytes,', Science 252 , 123-126 (1991).

${ }^{18}$ M. S. Jafri and J. Keizer, "On the roles of $\mathrm{Ca}^{2+}$ diffusion, $\mathrm{Ca}^{2+}$ buffers and the endoplasmic reticulum in $\mathrm{IP}_{3}$-induced $\mathrm{Ca}^{2+}$ waves,' Biophys. J. 69, 2139-2153 (1995).

${ }^{19}$ M. S. Jafri and J. Keizer, "Agonist-induced calcium waves in oscillatory cells: a biological example of Burger's equation,' Bull. Math. Biol. 59, 1125-1144 (1997).

${ }^{20}$ A. McKenzie and J. Sneyd, "On the formation and breakup of spiral waves of calcium,' Int. J. Bifurcation Chaos Appl. Sci. Eng. 8, 20032012 (1998).

${ }^{21}$ G. B. Ermentrout and N. Kopell, "Frequency plateaus in a chain of weakly coupled oscillators,', SIAM (Soc. Ind. Appl. Math.) J. Appl. Math. 15, 215-237 (1984).

${ }^{22}$ J. P. Keener and J. Sneyd, Mathematical Physiology (Springer-Verlag, New York, 1998).

${ }^{23}$ J. D. Murray, Mathematical Biology (Springer-Verlag, Berlin, 1989).

${ }^{24}$ D. Hansel, G. Mato, and C. Meunier, "Phase dynamics for weakly coupled Hodkin-Huxley neurons,', Europhys. Lett. 23, 367-372 (1993).

${ }^{25}$ N. Kopell and D. Somers, "Anti-phase solutions in relaxation oscillators coupled through excitatory interactions," J. Math. Biol. 33, 261-280 (1995).

${ }^{26}$ M. Kawato and R. Suzuki, "Two coupled neural oscillators as a model of the circadian pacemaker,' J. Theor. Biol. 86, 547-575 (1980).

${ }^{27}$ A. Sherman, "Anti-phase, asymmetric and aperiodic oscillations in excitable cells-I. Coupled bursters,'” Bull. Math. Biol. 56, 811-835 (1994).

${ }^{28} \mathrm{~V}$. Torre, "Synchronization of non-linear biochemical oscillators coupled by diffusion,'” Biol. Cybern. 17, 137-144 (1975).

${ }^{29}$ Y.-X. Li and A. Goldbeter, "Oscillatory isozymes as the simplest model for coupled biochemical oscillators,'” J. Theor. Biol. 138, 149-174 (1989).

${ }^{30}$ M. A. Taylor and I. G. Kevrekidis, "Some common dynamic features of coupled reacting systems,' Physica D 51, 274-292 (1991).

${ }^{31}$ D. G. Aronson, E. J. Doedel, and H. G. Othmer, "An analytical and numerical study of the bifurcations in a system of linearly-coupled oscillators,' Physica D 25, 20-104 (1987). 